OPEN ACCESS

Edited by:

Vincent Delorme

Institut Pasteur Korea, South Korea

Reviewed by:

Stacey Gilk,

Indiana University School of Medicine, United States

Simon Andrew Johnston University of Sheffield, United Kingdom

${ }^{*}$ Correspondence: Sharmistha Banerjee sbsl@uohyd.ac.in

Received: 30 June 2017 Accepted: 25 September 2017 Published: 10 October 2017

Citation: Asalla S, Mohareer K and Banerjee S (2017) Small Molecule Mediated Restoration of Mitochondrial Function Augments Anti-Mycobacterial Activity of Human Macrophages Subjected to

Cholesterol Induced Asymptomatic Dyslipidemia.

Front. Cell. Infect. Microbiol. 7:439.

doi: 10.3389/fcimb.2017.00439

\section{Small Molecule Mediated Restoration of Mitochondrial Function Augments Anti-Mycobacterial Activity of Human Macrophages Subjected to Cholesterol Induced Asymptomatic Dyslipidemia}

\author{
Suman Asalla, Krishnaveni Mohareer and Sharmistha Banerjee* \\ Molecular Pathogenesis Laboratory, Department of Biochemistry, School of Life Sciences, University of Hyderabad, \\ Hyderabad, India
}

Mycobacterium tuberculosis (M.tb) infection manifests into tuberculosis (TB) in a small fraction of the infected population that comprises the TB susceptible group. Identifying the factors potentiating susceptibility to TB persistence is one of the prime agenda of TB control programs. Recently, WHO recognized diabetes as a risk factor for TB disease progression. The closely related pathological state of metabolic imbalance, dyslipidemia, is yet another emerging risk factor involving deregulation in host immune responses. While high cholesterol levels are clinically proven condition for perturbations in cardiac health, a significant fraction of population these days suffer from borderline risk cholesterol profiles. This apparently healthy population is susceptible to various health risks placing them in the "pre-disease" range. Our study focuses on determining the role of such asymptomatic dyslipidemia as a potential risk factor for susceptibility to TB persistence. Macrophages exposed to sub-pathological levels of cholesterol for chronic period, besides impaired release of TNF- $\alpha$, could not clear intracellular pathogenic mycobacteria effectively as compared to the unexposed cells. These cells also allowed persistence of opportunistic mycobacterial infection by M. avium and M. bovis BCG, indicating highly compromised immune response. The cholesterol-treated macrophages developed a foamy phenotype with a significant increase in intracellular lipid-bodies prior to M.tb infection, potentially contributing to pre-disease state for tuberculosis infection. The foamy phenotype, known to support M.tb infection, increased several fold upon infection in these cells. Additionally, mitochondrial morphology and function were perturbed, more so during infection in cholesterol treated cells. Pharmacological supplementation with small molecule M1 that restored mitochondrial structural and functional integrity limited M.tb survival more effectively in cholesterol exposed macrophages. Mechanistically, M1 molecule promoted clearance of mycobacteria by reducing total cellular lipid content and restoring mitochondrial morphology and function to its steady state. We further supported our observations by infection assays in PBMC-derived macrophages from clinically healthy volunteers with 
borderline risk cholesterol profiles. With these observations, we propose that prolonged exposure to sub-pathological cholesterol can lead to asymptomatic susceptibility to M.tb persistence. Use of small molecules like M1 sets yet another strategy for host-directed therapy where re-functioning of mitochondria in cholesterol abused macrophages can improve M.tb clearance.

Keywords: dyslipidemia, Mycobacterium tuberculosis, mitochondria, small molecule M1, pre-disease, cholesterol, infection

\section{INTRODUCTION}

Tuberculosis (TB), caused by the bacillus Mycobacterium tuberculosis (M.tb), affects one-third of the global population, despite being completely curable with an unfortunate exception of totally resistant M.tb (World Health Statistics, 2017). Surprisingly, $<10 \%$ of immunocompetent people infected with M.tb develop the disease, while an astounding $90 \%$ successfully control the infection without showing any disease symptom, suggesting that only a minor fraction constitute the susceptible group (ATS, 2000). While issues like emergence of drug resistance, inability to identify latent cases and co-epidemic with HIV cloud effective TB management, yet another pressing need is to identify factors that are responsible for causing susceptibility to TB. Genetic susceptibility to TB has been long established in animal models demonstrating that genetic resistance or susceptibility to an infection can be bred into a population (Hoal, 2002). While malnutrition was always linked to TB susceptibility (Lonnroth et al., 2010; Cegielski et al., 2012), of late, lifestyle factors leading to dysglycemia, dyslipidemia, or even change in gut microbiota due to aberrant antibiotic use have been linked to M.tb susceptibility and survival (Khan et al., 2016). What seems to be of utmost importance is the innate immune response at the time of infection which decides if the bacteria will be eliminated or will survive in its niche, primarily alveolar macrophages. Clinical screenings have often identified conditions that range from health and disease, classifying the stages as "pre-disease." These pre-disease states, if can be intervened effectively may reduce the susceptibility, persistence and progression of $\mathrm{TB}$ infection to TB disease.

Diabetes and obesity have been associated with $\mathrm{TB}$ disease progression (Hanrahan et al., 2010; Kumar and Babu, 2017). However, the role of closely related pathological state of metabolic imbalance, dyslipidemia, in host immune response to infections has not been addressed adequately. Dyslipidemia, manifested by high levels of total cholesterol, is either cause or consequence of several pathological conditions, like Type 2 diabetes mellitus (T2DM), excessive alcohol consumption, liver diseases and nephrotic syndrome (Goldberg, 2001; Kronenberg, 2005; Katsiki et al., 2016). There are also rising clinical evidence that suggest that chronic levels of "borderline" high cholesterol can dramatically increase the risk of cholesterol associated complications by nearly $40 \%$ later in life (Nelson, 2013). Besides, TB is also known to cause both hyperglycemia and hypercholesterolemia in patients (Padmapriyadarsini et al., 2011). Recent reports have indicated that pathogen-induced dysregulation of host lipid synthesis and sequestering in macrophages leads to cholesterol-loaded macrophages, called "foamy macrophages" which are critical components in both bacterial survival and dissemination (Russell et al., 2009). These macrophages are characterized by increased total cellular lipids constituting cholesterol and triacylglycerols (TAGs). In other studies, hypercholesterolemia has been shown to cause the death of pancreatic $\beta$-cells thereby promoting diabetic like condition (Hao et al., 2007). Using a mouse model the relationship between hypercholesterolemia, mitochondrial damage and cardiovascular-tissue function was determined, where they concluded that cardiovascular disease risk factors- hypercholesterolemia and second hand smoke cause mitochondrial damage and dysfunction (Knight-Lozano et al., 2002). Exogenous treatment of cholesterol induced pancreatic $\beta$-cell apoptosis through oxidative stress (Lu et al., 2011). Accumulation of cholesterol in mitochondrial membrane interferes with oxidative phosphorylation and anti-oxidant defense that result in the generation of ROS which culminates in cell death (Bosch et al., 2011). Further, it was shown that cholesterol induced damage in pancreatic $\beta$-cells is channelized through mitochondrial dysfunction (Asalla et al., 2016). These studies clearly indicate that hypercholesterolemia can lead to mitochondrial dysfunction that can get manifested in various pathological conditions.

Mitochondrial dysfunction, besides impaired phagosome maturation and lysosomal storage disorders, is decisive in the outcome of macrophage infection by pathogenic mycobacteria (Duan et al., 2002; Berg et al., 2016). Mitochondrial damage disturbs the balance of pro- and anti-apoptotic factors during $M . t b$ infection, orienting the cell-death pathways to necrosis instead of apoptosis and helping mycobacterial dissemination. Specific M.tb factors like Rv2626c and Rv3873 (PPE68) have been implicated in promoting necrosis and dissemination (Danelishvili et al., 2016) while Rv2456c inhibited apoptosis (Jurcic Smith and Lee, 2016). Virulent mycobacteria, but not avirulent mycobacteria, have also been shown to induce significant variations to mitochondrial transmembrane potential, promoting necrosis (Chen et al., 2006). With these independent linkages between cholesterol, mitochondrial damage and mycobacterial pathogenesis, we speculated if cholesterol induced mitochondrial damage can lead to susceptibility to mycobacterial persistence.

While cardiac problems are apparently the most studied consequence of hypercholesterolemia, the metabolic linkages between cholesterol, diabetes and immune functions render a more complex situation. With these complex inter-relationships culminating into metabolic disorders, we sought to determine the 
role of asymptomatic dyslipidemia as a potential "pre-disease" condition and a risk factor for TB progression. Here we refer to the condition where individuals are clinically healthy but exhibit borderline risk cholesterol profile, that is, total cholesterol (TC): HDL ranging from 4.5 to 11 and LDL: HDL cholesterol ranging from 3 to 6 (Millán et al., 2009). Since such individuals are clinically healthy, these levels of cholesterol have been considered as sub-pathological. To address this, we established a cell culture based infection model where human macrophages were exposed to $80 \mu \mathrm{M}$ cholesterol that retained cell viability throughout the experiment mimicking sub-pathological cholesterol exposure. Using this model, we showed that macrophages exposed to subpathological cholesterol levels for the chronic duration, remained viable, but were highly susceptible to not only pathogenic mycobacteria like $\mathrm{H} 37 \mathrm{Rv}$ but also opportunistic mycobacteria like $M$. avium and $M$. bovis BCG. We further deciphered that this is mediated through mitochondrial dysfunction. We found that restoring these mitochondrial parameters to a steady state by pharmacological small molecule M1 reinstated the ability of intracellular mycobacterial clearance in cholesterol treated macrophages. We confirmed these inferences with PBMC derived macrophages from healthy volunteers showing borderline risk serum cholesterol profile. With this study, we propose that sub-pathological level of cholesterol is a "predisease" condition for TB persistence and small molecules like M1 hold the potential to be developed as a host-directed therapy for TB management.

\section{MATERIALS AND METHODS}

\section{Bacterial Cultures and Infections}

The pathogenic M.tb laboratory strain H37Rv, M. bovis BCG and M. avium (MTCC1723) were grown in Middlebrook 7H9 broth supplemented with 10\% OADC (Himedia), 0.5\% (v/v) glycerol and $0.05 \%(\mathrm{v} / \mathrm{v})$ Tween-80. The culture was checked for possible contamination using Ziehl-Neelsen $(\mathrm{ZN})$ staining procedure. Bacteria were grown in $7 \mathrm{H} 9$ broth with gentle shaking to an $\mathrm{OD}_{600}$ of 1.0 and harvested at $3,500 \times \mathrm{g}$ for $5 \mathrm{~min}$. The cells were then resuspended in RPMI without antibiotics and dispersed by passage through a $100 \mathrm{U}$ insulin syringe 10 times. The cells were counted following McFarland Nephelometer standards. MOIs were calculated based on these numbers and used for infection for $4 \mathrm{~h}$ at $37^{\circ} \mathrm{C}$ with $5 \% \mathrm{CO}_{2}$. The cells were washed $4 \mathrm{~h}$ post-infection thrice with PBS, followed by suspension in RPMI complete media with antibiotics and further incubated for the indicated time at $37^{\circ} \mathrm{C}$ with $5 \%$ $\mathrm{CO}_{2}$ and processed as per the experiments described. All the experiments involving mycobacterial strains were performed in BSL3 facility following appropriate biosafety protocols approved by Institutional Biosafety committee (IBSC No: SB-N-98).

\section{Cell Culture and PBMC Assays}

The infection assays and CFU enumeration assays were performed using either human monocytic cell line, THP-1, or PBMCs isolated from healthy volunteers.

The human monocytic cell line, THP-1 (ATCC) was maintained in RPMI 1640 medium supplemented with 10\% (v/v) FBS and differentiated using $25 \mathrm{ng} / \mathrm{mL}$ PMA overnight followed by a resting period of $10-12 \mathrm{~h}$. Subsequently, cholesterol treatment of differentiated THP-1 cells was carried out following the method described by Lu et al. with slight modifications. 250X ( $\sim 7 \mathrm{mM}$ ) Cholesterol Lipid Concentrate (CLC) (GIBCO, Grand Island, NY, USA) was diluted in RPMI and then added to THP1 cells at indicated time points and concentrations. In the case of infection, cells were pre-treated with cholesterol $(80 \mu \mathrm{M})$ for $12 \mathrm{~h}$ followed by infection for $4 \mathrm{~h}$ as described. Small molecule M1 (Sigma) treatment was given post-infection till the rest of the assay.

Peripheral Blood Mononuclear Cells (PBMCs) isolation was performed as per the protocol described by Panda et al. (2012). Fifteen milliliter of peripheral blood was taken from clinically healthy human volunteers $(N=7)$ after taking prior written consents from subjects as per approved guidelines of Institutional Ethics Committee (IEC). These volunteers were profiled for their total serum cholesterol (TC), HDL, and LDL cholesterol from an $\mathrm{NABL}^{1}$ (Govt. of India) accredited diagnostic lab. The PBMCs were isolated from heparinized blood by density gradient centrifugation method using Histopaque (Sigma). Briefly, the blood from each individual was diltuted twice with RPMI complete media and gently overlayed on Histopaque at 1:1 ratio followed by centrifugation at $100 \times \mathrm{g}$ for $30 \mathrm{~min}$. The buffy coat layer containing PBMCs was gently aspirated and transferred into sterile centrifuge tubes. The cells were harvested and washed with RPMI and further cultured in complete RPMI media supplemented with antibiotics. The cell number was quantified and adjusted to $0.2 \times 10^{6}$ cells/well in 24 well plates. The cells were cultured for 7 days at $37^{\circ} \mathrm{C}$. The non-adherent cells were eliminated by washing with RPMI media and the adherent cells were used for infections.

The various experimental categories included (i) cholesterol untreated uninfected; (ii) cholesterol treated uninfected; (iii) Cholesterol untreated infected; (iv) cholesterol treated infected; (v) cholesterol untreated uninfected, M1 treatment (vi) cholesterol untreated infected, M1 treatment (vii) cholesterol treated uninfected, M1 treatment and (viii) cholesterol treated infected, M1 treatment.

\section{CFU Enumeration of Viable Intracellular Bacilli}

For the CFU count, $0.2 \times 10^{6}$ THP- 1 cells were seeded per well in a 24 -well plate and infected with mycobacteria as per the experiments described. At the end of the experiment, infected cells were washed five times with PBS to remove any residual extracellular bacilli. The cells were then lyzed with sterile water at $37^{\circ} \mathrm{C}$ for $10 \mathrm{~min}$. The lysates were diluted and plated on $7 \mathrm{H} 10$ agar plates. The plates were incubated at $37^{\circ} \mathrm{C}$ with $5 \% \mathrm{CO}_{2}$ and 95\% humidity for 2-3 weeks and the colonies were enumerated.

Intracellular mycobacterial survival was also evaluated using Alamar Blue assays as described earlier (Ganji et al., 2016).

${ }^{1}$ NABL (National Accreditation Board for Testing and Calibration Laboratories).http://www.nabl-india.org/ 


\section{MTT Assay}

MTT assay was performed as per Vemula et al. (2016). Briefly, at the end of the experiment, MTT dye $(5 \mathrm{mg} / \mathrm{mL})$ was added at $25 \mu \mathrm{L}$ per $100 \mu \mathrm{L}$ media for $2 \mathrm{~h}$. The resulting formazan crystals were solubilized by $100 \mu \mathrm{L}$ of DMSO. The absorbance was measured at $540 \mathrm{~nm}$ using a multimode reader (BioTek).

\section{Estimation of Total Cellular Cholesterol Content in Macrophages}

THP- 1 cells were seeded in 6 well plates at a density of $2 \times 10^{6}$ cells/well and cultured overnight in complete medium. Following cholesterol treatment for indicated time, the amount of total cellular cholesterol in macrophage cell lysate was measured using commercial enzyme-based cholesterol assay kit (Cat\# STA-384 Cell Biolabs, Inc.) according to manufacturer's instructions.

\section{Oil Red O Staining}

Oil Red O staining was performed as per the protocol described in Ganji et al. (2016). The cells were washed with PBS, fixed with $10 \%$ formalin at room temperature for $30 \mathrm{~min}$. The cells were then washed with sterile water and incubated with $60 \%$ isopropanol for $5 \mathrm{~min}$, followed by staining with $60 \%$ Oil Red O Stain at $37^{\circ} \mathrm{C}$ for $5 \mathrm{~min}$ in the dark. The excess stain was washed thrice with sterile water. Intracellular lipid bodies were observed microscopically. The amount of lipid bodies were quantified by eluting the intracellular Oil Red O Stain using 100\% isopropanol at room temperature for $10 \mathrm{~min}$ and measuring the intensity at $500 \mathrm{~nm}$ spectrophotometrically with $100 \%$ isopropanol as blank.

\section{Analysis of Mitochondrial Membrane Potential (MMP) $(\Delta \psi M)$}

Mitochondrial membrane potential (MMP) was measured by JC1 staining kit (Cat. no. CS0390, Sigma) following manufacturer's instructions. $0.2 \times 10^{6}$ THP- 1 cells were seeded in 24 well plates and cultured overnight in complete medium. At the end of the experiment, cells from the different experimental categories described earlier were assayed. Briefly, the cells were washed with PBS and stained with $2.5 \mu \mathrm{M}$ of JC-1 in $1 \mathrm{X}$ JC-1 staining buffer. These cells were further incubated for $20 \mathrm{~min}$ at $37^{\circ} \mathrm{C}$ in a humidified atmosphere containing $5 \%$ $\mathrm{CO}_{2}$. Further, the cells were washed with ice-cold $1 \mathrm{X}$ JC-1 staining buffer. The cells were then resuspended in $0.5 \mathrm{ml}$ of the same buffer and analysis of the samples was performed using spectrofluorimetry (spectramax m5). JC-1 monomers were detected in the FL1 channel (green) and the aggregates were detected in FL2 channel (red), and the ratio of FL2/FL1 was calculated for determination of mitochondrial membrane potential.

\section{Detection of ADP and ATP Levels}

ATP and ADP levels were measured in cells of different experimental categories as described earlier by using ADP/ATP Ratio Assay Kit (Bioluminescent, ab65313), based on the bioluminescent detection, following manufacturer's instructions.

\section{Analysis of Mitochondrial ROS}

THP-1 cells at a density of $0.2 \times 10^{6}$ cells per well were seeded in 6 well plates and incubated overnight in complete medium. At the end of the experiment, cells from experimental categories, described in the text, were stained for 30 min with fluorogenic dye MitoSOX ${ }^{\mathrm{TM}}$ Red for mitochondrial ROS measurement following manufacturer's (Molecular Probes) instructions. Analysis of the samples was performed using spectrofluorimetry (spectramax m5).

\section{Confocal Microscopy}

$2 \times 10^{6}$ THP- 1 cells were seeded in six-well plates containing 20-mm-diameter glass coverslips and cultured overnight. At the end of the experiment, cells from various experimental categories were washed with PBS and stained with $50 \mathrm{nM}$ Mito-Tracker Red (M7512) in complete medium at $37^{\circ} \mathrm{C}$ for $30 \mathrm{~min}$, washed again with PBS and finally fixed with $4 \%$ paraformaldehyde. The coverslips were then mounted in Vectashield (Vector Laboratories), and images were visualized in confocal laser scanning microscope (Leica, Germany) with $63 \times$ oil immersion objective lens.

\section{Real-Time PCR}

Extraction of RNA from experimental cells was performed using trizol method as per manufacturer's instructions (Invitrogen). Two microgram of total RNA from each experimental category was used for reverse transcription using iScript cDNA Synthesis Kit as per manufacturer's protocol (BioRad) using 2X SYBR green mix (iTaq ${ }^{\mathrm{TM}}$ Universal SYBR Green Supermix, BioRad). GAPDH gene was used as endogenous control. The primers used for qRT-PCR are tabulated in Table S1. The real-time PCR data analyses were carried out using the $2^{-\Delta \Delta \mathrm{Ct}}$ method (Schmittgen and Livak, 2008).

\section{Enzyme Linked Immunosorbent Assay}

$2 \times 10^{6}$ THP-1 cells were seeded in six-well plates and followed by cholesterol treatment and infection. Cell culture supernatant was collected and filtered through $0.22 \mu \mathrm{m}$ syringe filter after infection and indicated time. For measurement of cytokine levels, BD OptEIA enzyme-linked immunosorbent assay (ELISA) kit was used following manufacturer's instructions. The absorbance was measured at 450 and $570 \mathrm{~nm}$ in multimode reader (BioTek). Each sample was assayed in triplicate.

\section{Transmission Electron Microscopy (TEM)}

The protocol was essentially followed as reported previously (Rajapakse et al., 2001) with minor modifications. Briefly, cells from each experimental category were fixed in $2.5 \%$ glutaraldehyde in $0.1 \mathrm{M}$ phosphate buffer $(\mathrm{pH} 7.4)$ for $24 \mathrm{~h}$ at $4{ }^{\circ} \mathrm{C}$ and washed with PBS twice each for $45 \mathrm{~min}$, then post-fixed in $1 \%$ aqueous Osmium Tetraoxide (OsO4) for $2 \mathrm{~h}$. Subsequently, the cells were washed with deionized distilled water for four times each for $45 \mathrm{~min}$, dehydrated in series of graded alcohols followed by infiltration and finally embedded in araldite 6005 
resin or spur resin. The cells were then incubated at $80^{\circ} \mathrm{C}$ for $48 \mathrm{~h}$ for complete polymerization. Ultra thin $(60 \mathrm{~nm})$ sections were made with a glass knife on an ultramicrotome (Leica Ultra cut UCT-GA-D/E-1/00), followed by mounting on copper grids and stained with saturated aqueous Uranyl acetate (UA) and counterstained with Reynolds lead citrate (LC). Sections were viewed under TEM (JEOL from Japan). Images were acquired digitally.

\section{Statistical Analysis}

Each experiment was carried out at the least three times (or more). The data are presented as mean $\pm S D$ (standard deviation). The statistical significance was assessed by $t$-test for the data distributed normally as inferred by normality test, for other data Mann-Whitney test was applied. This was performed using SigmaPlot version 11.0. A $p$-value of $\leq 0.05$ was considered statistically significant.

\section{RESULTS}

\section{Chronic Exposure to Sub-Pathological Levels of Cholesterol Impaired Macrophages from Clearing Intracellular Mycobacterium tuberculosis H37Rv}

With an aim to investigate the sequence of molecular events underlying the impact of long-term exposure (chronic) to subpathological levels of cholesterol on the clearance of intracellular pathogen $M . t b$ by human macrophages, we first developed a suitable model for mycobacterial infection wherein human THP1 monocytes, differentiated into macrophages using PMA, were infected with pathogenic mycobacteria $\mathrm{H} 37 \mathrm{Rv}$ during exposure to different levels of cholesterol for $64 \mathrm{~h}$ (Figure 1). A systematic titration of cholesterol concentration, cholesterol exposure time and MOI of M.tb infection was exercised ensuring THP-1 remained viable during the course of experiments. The cells were treated with increasing concentrations of cholesterol (i.e., $40,80,160$, and $250 \mu \mathrm{M}$ cholesterol) for $24 \mathrm{~h}$ and evaluated for viability by using tetrazolium salt (MTT) metabolite assay. We observed that up to $80 \mu \mathrm{M}$ of cholesterol, there was no effect on the viability of THP-1 while cholesterol concentration above $160 \mu \mathrm{M}$ showed significant cell death (see Figure S1A). It was further observed that cells remained viable up to $72 \mathrm{~h}$ upon $80 \mu \mathrm{M}$ cholesterol treatment, while $100 \mu \mathrm{M}$ cholesterol treatment decreased the cell viability significantly (see Figure $\mathrm{S} 1 \mathrm{~B})$. With these experiments, we concluded that $80 \mu \mathrm{M}$ of cholesterol was the maximum concentration of cholesterol that could be tolerated by THP-1 cells for $48 \mathrm{~h}$ post-infection in our experimental conditions. Hence, treatment with $80 \mu \mathrm{M}$ of cholesterol was subsequently used to represent borderline risk cholesterol (from now on referred to as sub-pathological cholesterol). After precise titrations of $\mathrm{H} 37 \mathrm{Rv}$ infections, we observed that cholesterol treated THP-1 cells could withstand $\mathrm{H} 37 \mathrm{Rv}$ infection at MOI 20 for $48 \mathrm{~h}$ (see Figure S1C), which was used for further studies. In summary, in our model, THP-1 cells were pre-treated with $80 \mu \mathrm{M}$ cholesterol for $12 \mathrm{~h}$ followed by mycobacterial infection (MOI 20) for $4 \mathrm{~h}$ and were kept in sustained presence of $80 \mu \mathrm{M}$ cholesterol for $48 \mathrm{~h}$ post-infection. The total exposure time of cholesterol in our experimental setup was $64 \mathrm{~h}$, after which assays were carried out, (Figure 1A). Since cells remained viable throughout the experimental period, these simulated conditions where cells exposed to "borderline risk cholesterol" were "asymptomatic and apparently healthy".

Using this model, the efficacy of these macrophages to eliminate intracellular $\mathrm{H} 37 \mathrm{Rv}$ was then evaluated. THP-1 cells were infected with $\mathrm{H} 37 \mathrm{Rv}$ in the presence and absence of cholesterol and live intracellular $\mathrm{H} 37 \mathrm{Rv}$ were scored through CFU. We observed significantly high CFU counts from THP-1 treated with cholesterol, showing $1790 \pm 192.0 \mathrm{CFU}$ as against $991.7 \pm 94.35 \mathrm{CFU}$ seen from untreated THP-1 (Figure 1B). Thus, there was an increase in live persistent bacteria by $80.5 \%$ in cholesterol-treated cells. Since some of the earlier cell culture-based studies have implicated the role of cholesterol in supporting entry of intracellular pathogens like Brucella and M. bovis into macrophages (Gatfield and Pieters, 2000; Naroeni and Porte, 2002; Viswanathan et al., 2015), we monitored mycobacterial CFU in treated and untreated THP- 1 cells at $0 \mathrm{~h}$ post-infection (see Figure S1D). The results showed that there was an insignificant difference in mycobacterial CFU at $0 \mathrm{~h}$ in both treated $(1,788 \pm 411.5 \mathrm{CFU})$ and untreated cells $(1,705$ $\pm 271 \mathrm{CFU})$ clearly showing that cholesterol concentration and exposure time in our model did not affect mycobacterial entry. This suggested that intracellular events post-mycobacterial entry may have supported mycobacterial survival in the cholesterol exposed THP-1 cells.

Having observed that $64 \mathrm{~h}$ of sub-pathological cholesterol exposure impaired clearance of pathogenic mycobacteria by otherwise viable macrophages (Figure 1B), we checked the levels of intracellular cholesterol and lipid bodies in these cells. Recent studies have pointed that, macrophages with accumulated lipid bodies (foamy macrophages) contribute to tissue pathology and exhibit limited protection against persistent bacteria (Russell et al., 2009; Daniel et al., 2011). At the same time, pathogeninduced dysregulation of host lipid synthesis and requisitioning have been shown to impair protective immunity against TB in a mouse model (Martens et al., 2008). While such observations have been made with high cholesterol treatments, we observe that even chronic exposure to sub-pathological cholesterol levels resulted in the accumulation of cholesterol in macrophages. Intracellular lipid and total cellular cholesterol concentrations were evaluated as described in methods. Both microscopic examination (Figure 1C) and spectrophotometric quantification (Figure 1D) showed that cholesterol pre-treatment for $12 \mathrm{~h}$ in THP-1 cells resulted in an increase of intracellular lipid bodies by $32.7 \%$ and $90.5 \%$ when sustained for next $48 \mathrm{~h}$ (please refer Figure 3B), giving these cells a sustained "foamy" phenotype during the course of the experiment.

We then investigated the potential of these cells to release TNF- $\alpha$, a potent pyrogenic cytokine that stimulates the acute phase of the immune response and is also one of the first cytokines to be released in response to a pathogen (Beutler, 1999). TNF- $\alpha$ plays a key role both in initial and chronic 


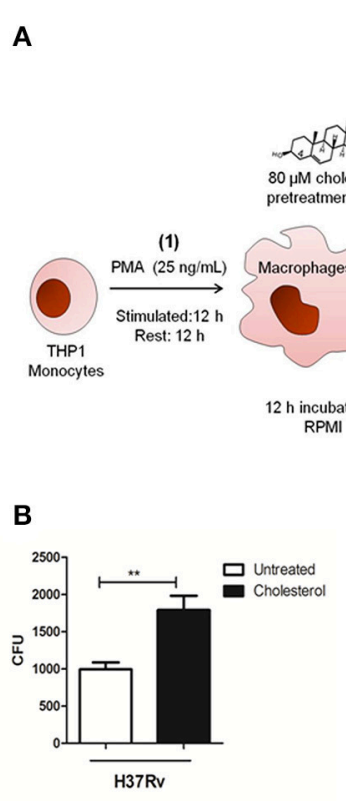

E

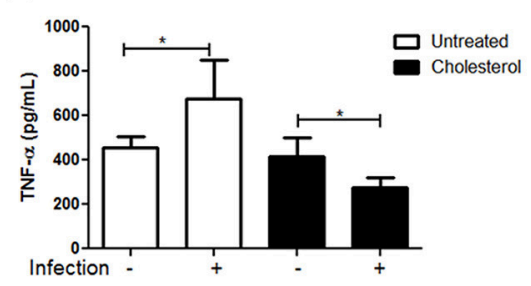

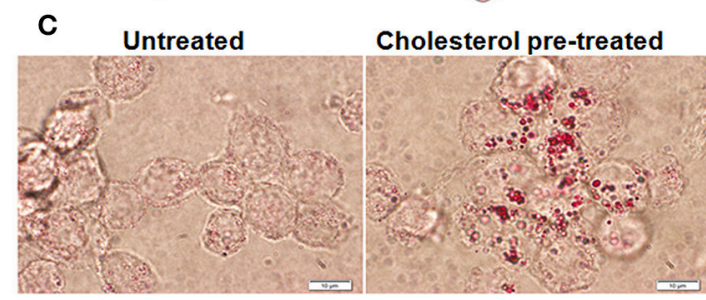

Cholesterol pre-treated

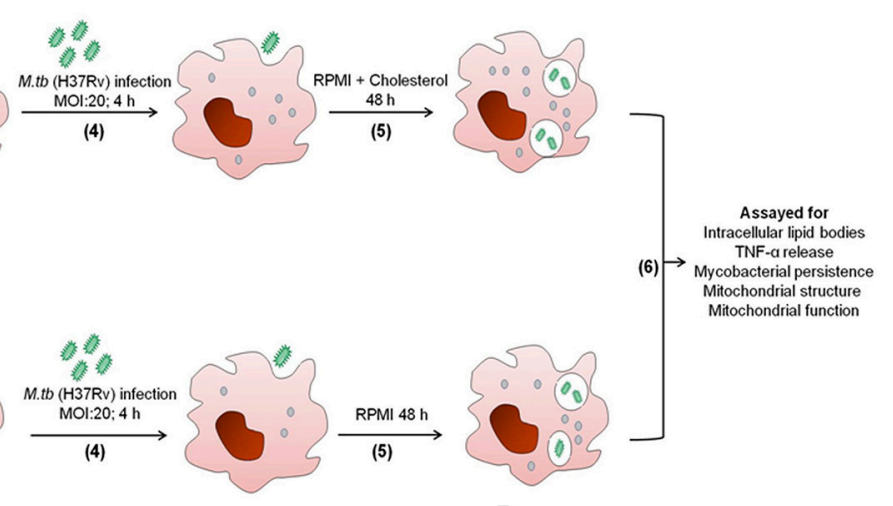

D

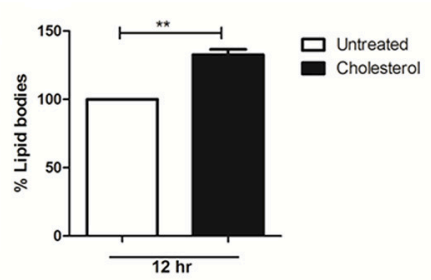

F

G

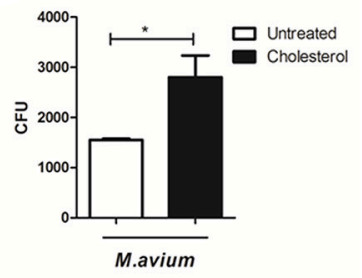

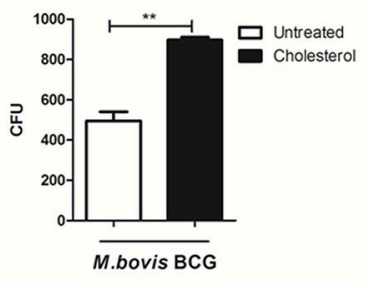

FIGURE 1 | Chronic exposure of THP-1 cells to sub-pathological levels of cholesterol impaired them from clearing intracellular Mycobacteria. (A) Schematic representation of the cell culture based model used in the study (B) Measurement of the clearance of intracellular pathogenic mycobacteria H37Rv by CFU counts in untreated and treated cells. (C) Oil Red O staining of intracellular lipid bodies in untreated and treated cells $12 \mathrm{~h}$ post cholesterol ( $80 \mu \mathrm{M})$ treatment before infection. The bright field images for each category are representative of at the least five fields (scale bar $10 \mu \mathrm{m}$ ). (D) Lipid bodies as visualized in (C) were quantified on microtiter plate spectroscopically after Oil Red O staining. (E) TNF- $\alpha$ released by untreated and cholesterol treated, with (MOI 20) or without H37Rv infection as measured by ELISA. Clearance of (F) non-tuberculous mycobacteria $M$. avium or (G) attenuated mycobacteria $M$. bovis BCG by untreated or treated THP-1 cells as evaluated by CFU. Each experiment was carried out at the least three times. Statistical analyses were performed as mentioned in methods. Error bars represent $\pm S D .{ }^{\star} P \leq 0.05 ;{ }^{* *} P<0.005$

phase of M.tb infection (Lin et al., 2007). As reported earlier, we observed an induction of TNF- $\alpha$ secretion in response to $\mathrm{H} 37 \mathrm{Rv}$ infection (1.48-fold increase as compared to unifected, untreated). However, it was observed that both treated and untreated THP-1 cells released a similar amount of TNF- $\alpha$ (Treated $417.7 \pm 37.17$ and untreated $454.5 \pm 22.92 \mathrm{pg} / \mathrm{mL}$ ). But upon $\mathrm{H} 37 \mathrm{Rv}$ infection, the treated cells failed to release adequate levels of TNF- $\alpha(276.5 \pm 19.94 \mathrm{pg} / \mathrm{mL})$ as compared to untreated infected cells $(675.6 \pm 87.86 \mathrm{pg} / \mathrm{mL})$ (Figure 1E). Taken together, these experiments showed that while cholesterol exposed cells were viable, they failed to respond appropriately to mycobacterial infection.

Having observed a compromised protective response by cholesterol treated infected cells, we hypothesized that such microenvironment could make macrophages susceptible to persistent infections even by opportunistic mycobacteria. To test the same, we infected treated and untreated THP-1 cells with non-tuberculous pathogenic mycobacteria (NTM) M. avium and attenuated mycobacteria, $M$. bovis BCG. We observed that as compared to untreated $(1,550 \pm 28.87 \mathrm{CFU})$, there was an increase of $80.64 \%$ of persistent $M$. avium $(2,800 \pm 435.9$ CFU) in cholesterol treated THP-1 cells (Figure 1F), while for $M$. bovis BCG it was observed to be increased by $81.2 \%$ (untreated $495.0 \pm 45.37 \mathrm{CFU}$ and treated 897.0 $\pm 13 \mathrm{CFU}$ ) (Figure 1G). The increase in persistent bacteria inside cholesterol treated THP-1 cells indicated that these cells are relatively immune-compromised and provide a conducive environment to these opportunistic mycobacteria for their growth and survival. Collectively, with these experiments, we conclude chronic exposure of macrophages to sub-pathological cholesterol creates a conducive environment in the form of foamy phenotype, setting a "pre-disease" state in the cells supporting M.tb persistence. We further investigated the molecular events underlying the same using this model. 


\section{Chronic Exposure to Sub-Pathological Cholesterol Impaired Mitochondrial Function in Infected Macrophages Which Could Be Rescued Using Small Molecule M1}

Some of the earlier assays with exogenous treatment of cholesterol suggested mitochondrial dysfunction resulting in apoptotic cell death (Lu et al., 2011). While most of these studies advocated that it leads to activation of apoptosis, our model clearly has taken conditions where cell death is minimal upon exogenous treatment of cholesterol.

To check if sub-pathological cholesterol can increase the risk of mitochondrial malfunction upon infection, we first studied alterations in mitochondrial structure microscopically. For this, mitochondrial morphology was monitored using fluorescent staining and transmission electron microscopy (TEM). Fluorescent staining of mitochondria from untreated and treated THP-1 cells, with or without infection, using MitotrackerRed showed distinct morphological differences (Figure 2A). In the control cells, the majority of mitochondria were observed to be rod-shaped, elongated, and forming interconnected structures, while infected cells showed predominantly nod-shaped, ovoid and solitary mitochondria, indicating stress morphology (Figure 2A, upper panel, columns i and ii). Such stress morphology could be observed in cholesterol exposed THP-1 cells even before infection, which augmented several fold upon infection where solitary spheres and rods of various sizes were predominantly seen (Figure 2A, lower panel, column $\mathrm{i}$ and ii). These observations were also supported by TEM imaging (Figure 2B). Transmission electron micrographs clearly show that mitochondrial morphology in control cells was predominantly elongated rod-shaped and interlinked, while the same in either cholesterol treated or infected cells were predominantly ovoid nod-shaped and solitary (Figure 2B, lower panel column i and upper panel column ii, respectively). Infection of cholesterol treated THP-1 cells resulted in a more noticeable change in mitochondrial morphology where mitochondria appeared swollen and spherical (Figure 2B, lower panel column ii). Additionally, we also looked for associated changes in nuclear morphology using confocal microscopy, where the images were focused on nuclei (Figure S5). We did not observe any changes in nuclear morphology. With these visual evidences, we could conclude that exposure to sub-pathological levels of cholesterol leads to changes in mitochondrial morphology, very similar to that caused by M.tb infection alone. This indicated that cholesterol exposed cells are already under stress, which gets several fold amplified upon infection as evident from swollen mitochondrial morphology.

To assess the mitochondrial structure in relation to changes in the expression of genes involved in mitochondrial dynamics, mRNA levels of mitochondrial fusion and fission genes $m f n-1$, $m f n-2, d r p-1$, and $m f f$ in these macrophages were measured through qRT-PCR (Figures 2C-F). The levels of $m f n-1$ and $m f n-2$ were significantly decreased by $\sim 2$-fold in cholesterol treated infected cells, whereas $m f n-2$, but not $m f n-1$, was downregulated by $\sim 2$-fold during infection in untreated cells

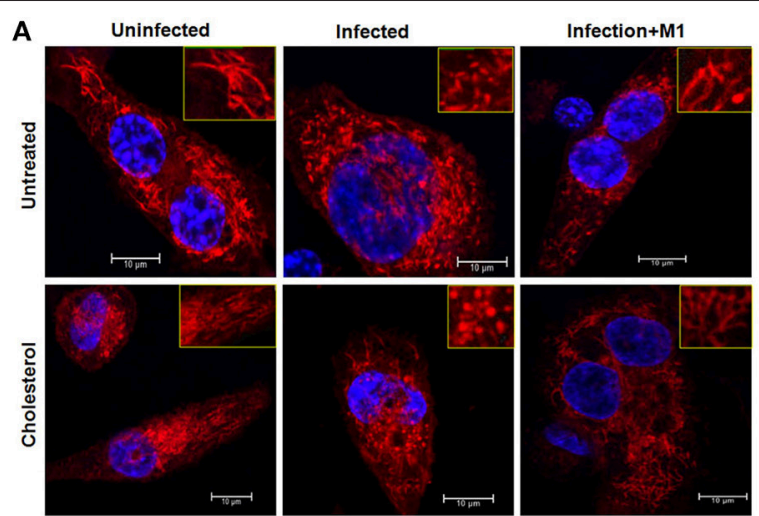

B

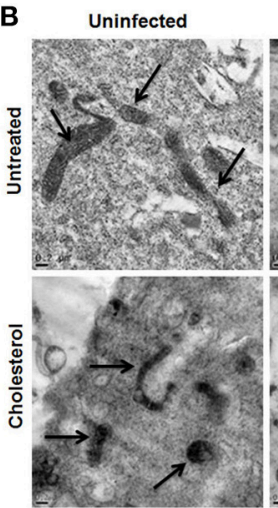

C

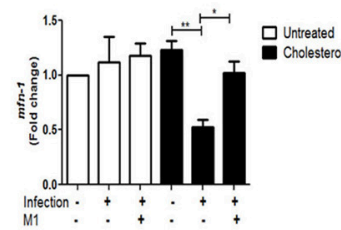

E

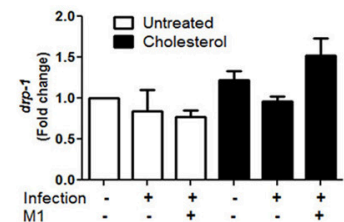

G

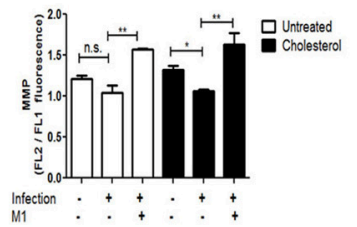

Infected

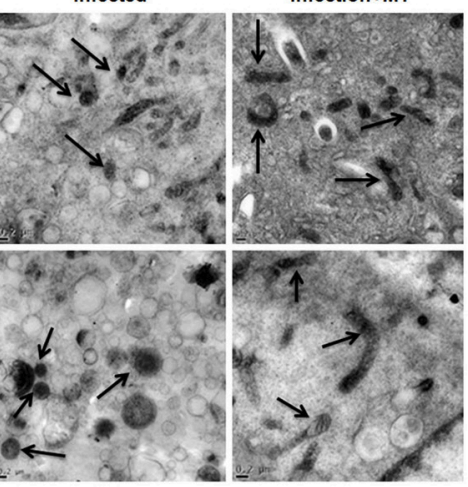

D

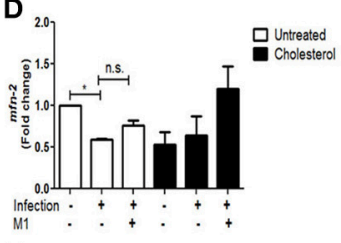

$\mathbf{F}$
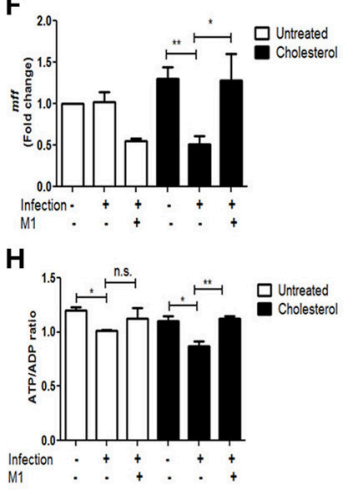

FIGURE 2 | Mycobacterial infection in cholesterol treated THP-1 cells altered mitochondrial structure and function, which was rescued by small-molecule M1. (A) Confocal microscopy showing distribution and shape of mitochondria stained by mito-tracker red (seen in red fluorescence) against a background of DAPI stained cells from each category. The images are representative of at the least five fields (scale bar $10 \mu \mathrm{m}$ ). The inset in each image is the zoomed image showing the gross mitochondrial shape. (B) Transmission electron micrographs depicting changes in the mitochondrial morphology from cells from each category. Arrows indicate representative mitochondria. The bar represents $0.2 \mu \mathrm{m}$ scale. qRT-PCR-based changes in mRNA expression levels

(Continued) 
FIGURE 2 | Continued

of mitochondrial fusion genes $m f n-1$ (C) and $m f n-2$ (D) as well as mitochondrial fusion genes $d r p-1(\mathbf{E})$ and $m f f(\mathbf{F})$ in each category. Fold change in transcript levels were calculated as against untreated and uninfected THP-1 cells. The transcript levels were quantified by relative $2^{-\Delta \Delta C t}$ normalized against endogenous GAPDH. (G) Measurement of Mitochondrial Membrane Potential in each category as described in methods. The data is expressed as the ratio of FL2 to FL1. $\mathbf{( H )}$ Graphical representation of intracellular ATP/ADP ratios in each category using bioluminescence assay as described in methods. Each experiment was carried out at the least three times. Statistical analyses were performed as mentioned in methods. Error bars represent $\pm \mathrm{SD} .{ }^{*} P \leq 0.05 ;{ }^{\star \star} P<0.005$.

(Figures 2C,D). The levels of $d r p-1$ did not change significantly in any condition (Figure 2E). However, levels of $m f f$ were observed to be significantly reduced by $\sim 2$-fold in cholesterol treated infected cells as compared to cholesterol treatment alone (Figure 2F). Overall, the changes in the expression levels of mitochondrial fission and fusion genes support the variations in the mitochondrial structural dynamics as observed microscopically.

With the clear suggestion of changes in mitochondrial structure, we further studied the changes in mitochondrial function. MMP in untreated and cholesterol treated THP1 cells, with and without infections were measured using fluorometric assays (Figure 2G). It was observed that while either infection or cholesterol alone did not significantly affect MMP, infection of cholesterol-treated cells caused a consistent (20.07\%) reduction in MMP. Since the change in MMP should reflect in ATP production, in addition to this, mitochondrial function was assayed by measuring ATP/ADP ratio. ATP/ADP ratio in untreated infected cells was $15.65 \%$, while that in treated infected cells was $21.08 \%$, suggesting cumulative damage to mitochondria upon cholesterol treatment and infection together (Figure 2H). With these experiments, we registered a consistent change in mitochondrial functional parameters. Such subtle and consistent changes in mitochondria have been reported to have huge physiological consequences (Buckman and Reynolds, 2001) and should not be considered inconsequential. MitoROS production, upon infection of both untreated and treated cells, however, remained unaltered (please see Figure S2A). Further, as control experiments, to check if cholesterol concentrations $<80 \mu \mathrm{M}$ could cause alterations in mitochondrial structure and function, $20 \mu \mathrm{M}$ and $40 \mu \mathrm{M}$ cholesterol treatments were used to assay for their impact on both mitochondrial structure and function (Figures S2B,C) and clearance of mycobacteria (Figure S2D). We do not observe any significant change in mitochondrial morphology or function, neither any differences in mycobacterial clearance upon treatment with 20 and $40 \mu \mathrm{M}$ cholesterol as compared to the untreated.

These results implicated that, prolonged exposure to subpathological cholesterol levels created an amicable environment for M.tb in otherwise apparently healthy macrophages, by compromising mitochondrial structure and function, which significantly decreases the anti-mycobacterial activity of infected cells, thereby promoting mycobacterial persistence.
We next attempted to restore the mitochondrial activity using a commercially available, pharmacological small molecule, M1, which has been reported to restore mitochondrial health in various systems upon different stresses (Wang et al., 2012). We standardized the effective concentration of M1 in our experimental conditions, keeping the cells viable (please see Figure S3A). THP-1 cells, untreated and cholesterol treated, with and without $\mathrm{H} 37 \mathrm{Rv}$ infections, were treated with $30 \mu \mathrm{M}$ of M1 post-infection for $48 \mathrm{~h}$. All the mitochondrial parameters, viz; morphology, expression of genes responsible for mitochondrial dynamics, MMP and ATP/ADP ratio were reanalyzed in the presence of M1. We observed that mitochondrial morphology was substantially restored upon M1 treatment of both untreated and treated infected cells (Figure 2A lower panel column iii and Figure 2B lower panel column iii, respectively). M1 also restored $m f n 1$ and $m f n 2$ levels in cholesterol treated infected cells, but not in untreated infected cells (Figures 2C,D respectively). We, for the first time, observed that M1 restored levels of the fission gene $m f f$ in cholesterol treated infected cells (Figure 2F). The restoration of the expression levels of these genes, supported by re-establishment of morphological features, suggested that M1 probably reinstated the balance between fission and fusion events, and this equilibrium maintained the steady state mitochondrial dynamics, more efficiently in cholesterol-treated cells. We also observed that consequent restoration of MMP (Figure 2G) and ATP/ADP ratio (Figure 2H) by M1 during infection of cholesterol exposed cells.

\section{Rescuing Mitochondrial Function and Reduction of Intracellular Lipid Bodies by M1 Increased Mycobacterial Clearance in Cholesterol Treated Infected Macrophages}

We next postulated if restoring mitochondrial structure and function in cholesterol exposed THP-1 by M1 will potentiate these cells for mycobacterial clearance. Having noted that M1 treatment rescued mitochondrial function in cholesterol treated THP-1, we checked the lipid bodies and intracellular cholesterol levels in these cells. In compliance with other studies, we observed that $\mathrm{H} 37 \mathrm{Rv}$ infection alone resulted in the increase in lipid bodies by $53.77 \%$ (Figure 3A upper panel column ii and Figure 3B). This impact was more pronounced in cholesterol treated H37Rv infected cells which showed an increase in intracellular lipids by $76.2 \%$ as compared to infection in untreated cells (Figure 3A lower panel column ii and Figure 3B).

Besides restoring mitochondrial structure and function, when cholesterol exposed infected cells were treated by M1, it resulted in a decrease in the intracellular lipid bodies by $58 \%$ (Figure 3A lower panel column ii and iii and Figure 3B), as well as total intracellular cholesterol levels by 15\% (Figure 3C). M1 treatment could not reduce either lipid bodies or cholesterol significantly in untreated infected cells (Figure 3A upper panel column ii and iii, Figures 3B,C). Finally, the impact of M1 on mycobacterial clearance was scored. Pharmacological intervention by M1 reduced the intracellular $\mathrm{H} 37 \mathrm{Rv}$ burden of cholesterol treated infected cells by $46.16 \%$ and of untreated infected cells by $27.87 \%$. This indicated that the efficacy of M1 in clearance 
A

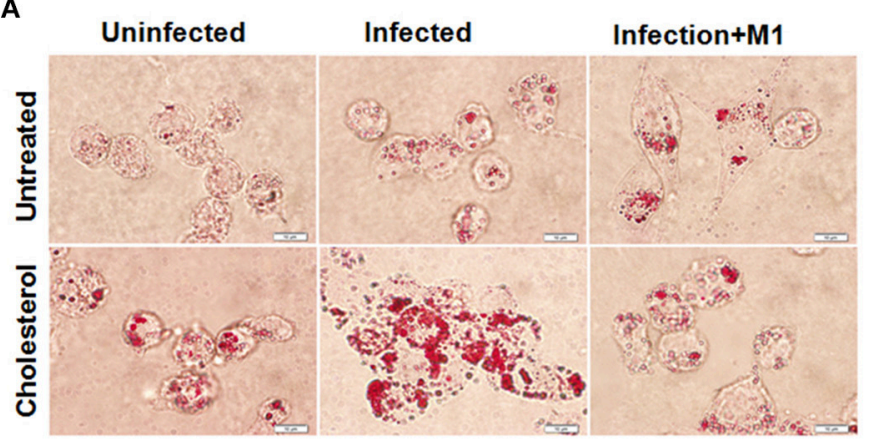

B

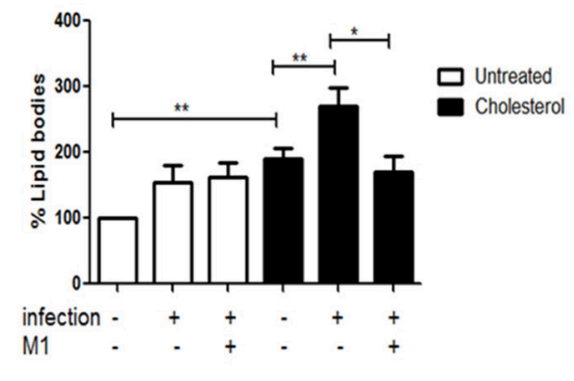

D

C
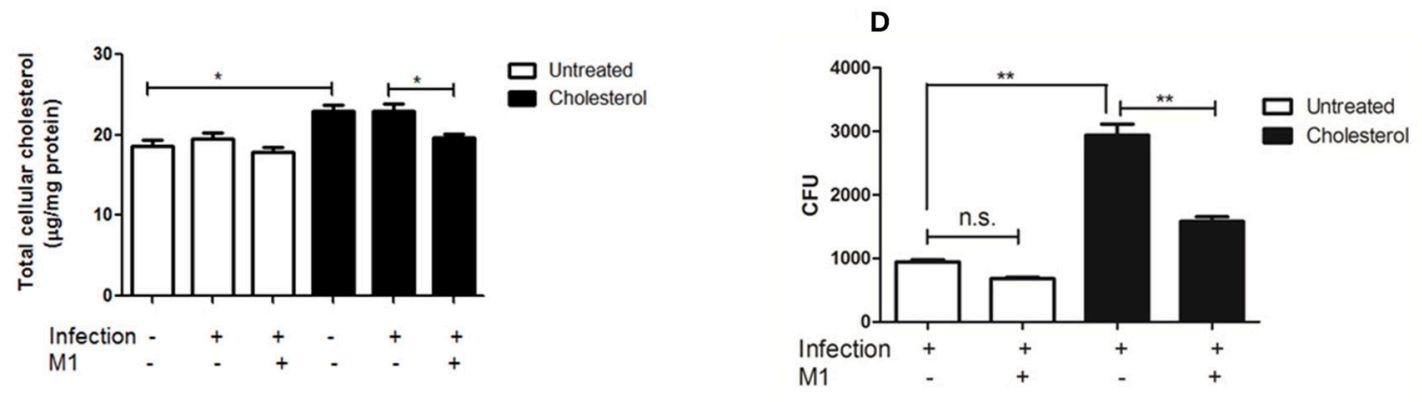

FIGURE 3 | Pharmacological intervention of M1 improves mycobacterial clearance by cholesterol treated THP-1 cells. (A) Representative bright field microscopy image showing the accumulation of lipid bodies by Oil Red O staining inside the untreated or cholesterol treated H37Rv infected cells upon $48 \mathrm{~h}$ post-infection, with or without M1 treatment. The white bar indicates $10 \mu \mathrm{m}$ scale. Images were taken at $100 \times$ magnification. (B) Bar graph representing the fold change in the accumulation of Oil Red O Stain as measured at Abs500 nm in different categories. Percentage changes were calculated with respect to the values of the untreated uninfected THP-1 cell. (C) Colorimetric assay quantifying total cellular cholesterol in each category. (D) Measurement of H37Rv clearance by CFU counts upon M1 treatment of untreated and cholesterol treated infected THP-1 as described. Each experiment was carried out at the least three times. Statistical analyses were performed as mentioned in methods. Error bars represent $\pm \mathrm{SD}$. ${ }^{\star} P \leq 0.05 ;{ }^{\star \star} P<0.005$.

of mycobacterial infection was higher in cholesterol treated macrophages (Figure 3D). It is to be noted that M1 treatment of cholesterol untreated and treated cells in the absence of infection had insignificant impact on intracellular lipid and MMP (Figures S3B-D). It is also to be noted that M1 had no impact on the in-vitro growth of $\mathrm{H} 37 \mathrm{Rv}$ cultures (please see Figure S4).

\section{PBMC Derived Macrophages from Subjects with Borderline Risk Cholesterol Profile Showed Reduced Clearance of Intracellular Mycobacteria}

From the above experiments, we could infer that subpathological long term exposure to cholesterol impairs macrophages from adequately responding to mycobacterial infection and prevents its clearance. The small molecule M1 could rescue cholesterol exposed infected macrophages and helped in improving mycobacterial clearance. To validate these cell line based observations in primary cells, we used PBMC derived macrophages from human volunteers with either normal (referred to as NC) or borderline risk (referred to as BLC) cholesterol profiles (Table S2). Mycobacterial clearance was assayed by $\mathrm{CFU}$ enumeration from PBMC derived macrophages subjected to different conditions as in the chronic cell culture model (Figure 4). Similar to observations from the cell culture model, we found that intracellular clearance of mycobacteria by cholesterol treated PBMC derived macrophages from NC (139.8 \pm 22.84$)$ was significantly reduced $(46.3 \%)$ as compared to untreated cells $(64.76 \pm 9.29)$ (Figure 4A). We then compared the clearance of mycobacteria by PBMC derived macrophages from NC and BLC. As expected, PBMC derived macrophages from BLC (108.7 \pm 20.21$)$ showed reduced clearance $(61.3 \%)$ of mycobacteria as compared to those from NC $(64.76 \pm 9.29)$ (Figure 4B). Further, there was no difference in clearance of mycobacteria from PBMC derived macrophages of BLC either cholesterol treated $(119.9 \pm 17.84)$ or untreated $(108.7 \pm 20.21)$ (Figure 4C). Also, there was no significant difference in the clearance of mycobacteria from cholesterol treated PBMC derived macrophages from either NC $(139.8 \pm 22.84)$ or BLC $(119.9 \pm 17.84)$ (Figure 4D). These experiments showed that exogenously treated PBMC derived macrophages from $\mathrm{NC}$ behaved similar to those of untreated BLC confirming that PBMC derived macrophages from BLC had reduced efficiency of mycobacterial clearance.

M1 treatment did not improve the clearance of mycobacteria significantly from PBMC derived macrophages from either NC or BLC. However, when we compared improvement of clearance of mycobacteria between untreated NC and BLC, we found that while M1 could improve clearance in NC by $12.9 \%$, the same in BLC was $25.6 \%$ (Figure 4E). On the other hand, impact of M1 on mycobacterial clearance was more efficient in cholesterol 
treated PBMC derived macrophages from both NC and BLC (Figure 4F) reiterating that M1 is more effective in improving clearance from cholesterol exposed macrophages. These results supported that chronic exposure to borderline risk cholesterol created a favorable environment for $M . t b$ persistence.

In summary, we concluded that prolonged exposure to sub-pathological levels of cholesterol leads to increase in intracellular lipid bodies, alteration in mitochondrial structure and function that supported persistence of H37Rv and opportunistic pathogens. Pharmacological intervention by M1 reversed changes in cholesterol treated infected macrophages, thereby increasing the clearance of persistent mycobacteria (Figure 5).

\section{DISCUSSION}

Our study focuses on the role of asymptomatic dyslipidemia as a potential risk factor for tuberculosis progression and the cellular mechanism involved therein. We established a chronic cholesterol model to demonstrate the effect of sub-pathological concentration of cholesterol on the immune response to M.tb infection by human macrophages, categorizing the condition as a "pre-disease" state. A "pre-disease" is a designated precondition in an asymptomatic individual, identifying which may help to avert major health issues (Viera, 2011). As per global diagnostics standards, serum cholesterol is measured as total cholesterol (TC), low density (LDL), high density (HDL) cholesterol, where a ratio of TC/HDL ranging from 4.5 to 11 is designated as average to moderate risk and a ratio of $\mathrm{LDL} / \mathrm{HDL}$ cholesterol ranging from 3 to 6 is designated as borderline risk (NABL). Unfortunately, owing to lifestyle changes, much fraction of the population is shifting toward borderline risk. These apparently healthy populations are susceptible to various health risks. TB disease progression is contingent on the physiological background of the host (BoseDasgupta and Pieters, 2014). Over the years, researchers have attempted to identify conditions helping sustained persistence of TB or development of active TB disease from asymptomatic latent cases. While conditions like HIV infections are well-known to increase susceptibility to both fresh $M$.tb infection and activation of latent TB, in this study we, for the first time, showed, that chronic, asymptomatic dyslipidemia in the host can influence the fate of TB persistence and disease progression.

Towards this, we studied immune-competence of macrophages exogenously exposed to sub-pathological levels of cholesterol. The central point of the pre-disease model was a careful titration of cholesterol which will represent the "borderline risk" concentration of cholesterol for macrophages in cultures. We finally found that exposing macrophages to $80 \mu \mathrm{M}$ of cholesterol for $72 \mathrm{~h}$ did not reduce cell viability. This simulated the condition of "borderline risk" cholesterol treatment where cells remained pathologically asymptomatic and viable. The experiments clearly showed that though the cholesterol exposed cells were viable, they failed to exhibit a normal innate immune response, recorded in terms of TNF- $\alpha$ release (Figure 1E) and could not clear the intracellular M.tb load efficiently
(Figure 1B). Further, we also observed that these cells had dense intracellular lipid bodies upon infection (Figure 3A), exhibiting dysregulated lipid metabolism, giving "foamy" character to these macrophages. Such macrophages were also susceptible to persistent infection by NTM and attenuated mycobacteria, as shown by increased persistence of M. avium (Figure 1F) and M. bovis BCG (Figure 1G), which are otherwise cleared by untreated macrophages. Deregulation of lipid metabolism in host is known to contribute to the virulence mechanisms of M.tb. $M . t b$ infection causes perturbations in LXR and PPAR signaling pathways or cholesterol biosynthesis resulting in accumulation of lipid bodies. Several enzymes related to lipid metabolism are up-regulated mediated by PPAR $\gamma$ and LXR transcriptional regulators (Lovewell et al., 2016). The lipid bodies are consumed by the bacteria resulting in their expansion. This enormous requirement of lipid source is mainly because of the lipid-rich cell wall of mycobacteria. Recent studies identified cholesterol as an essential lipid for mycobacterial infection (Larrouy-Maumus, 2015). Additionally, Mce4 transport system of M.tb is shown to be essential for cholesterol import into bacterial cells, and genetic perturbation of mce4 locus results in severe attenuation in a mouse model of M.tb infection (D'Avila et al., 2006; Pandey and Sassetti, 2008; Almeida et al., 2009; Lee et al., 2013).

Some of the earlier experiments have established the role of cholesterol in M.tb entry. They noted cholesterol specific sensors present on the cholesterol-rich membrane domains of the host cells (Kaul et al., 2004) and accumulation of cholesterol at the site of bacterial entry (Gatfield and Pieters, 2000). Further, Viswanathan et al. showed that brief exposure to very high cholesterol treatment $(1 \mathrm{mM})$ facilitated M.tb entry through these lipid rafts in macrophage cell lines (Viswanathan et al., 2015). In contrast to these studies, we used much less concentration of cholesterol with a prolonged exposure time in our model. This was primarily keeping the objective of understanding the role of chronic exposure of subpathological cholesterol on M.tb infection. We confirmed that our conditions though did not support M.tb entry, assisted increased persistence of intracellular mycobacteria, besides making the cholesterol exposed macrophages susceptible to persistent infection by opportunistic mycobacteria. This could be linked to impaired immune function, dysregulated lipid accumulation and mitochondrial dysfunction in cholesterol treated macrophages. Treatment of these cells with a small molecule M1 reinstated the ability to clear intracellular mycobacteria in these macrophages by restoring mitochondrial structure and function besides reducing intracellular lipid bodies (Figure 5).

Moderate levels of cholesterol lead to mitochondrial dysfunction by causing structural and functional aberrations (Asalla et al., 2016). Mitochondria are poorly constituted with cholesterol in their lipid bilayers as compared to that of the plasma membrane. However, the low level of cholesterol in the mitochondrial inner membrane (MIM) favors important physiological functions including synthesis of bile acids in hepatocytes, maintenance of intact mitochondrial membrane structure, homeostatic membrane potential, OXPHOS and so on. In pathological conditions, accumulated cholesterol in MIM alter 
A

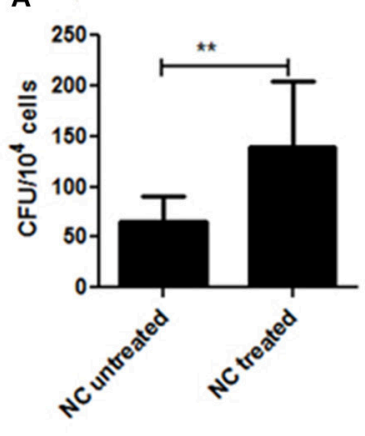

B

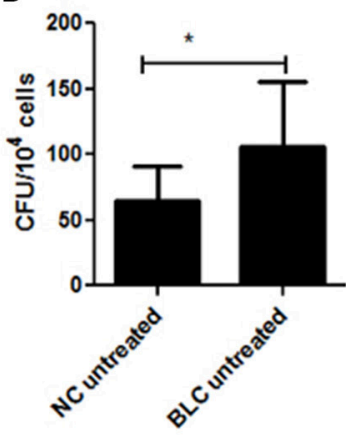

C

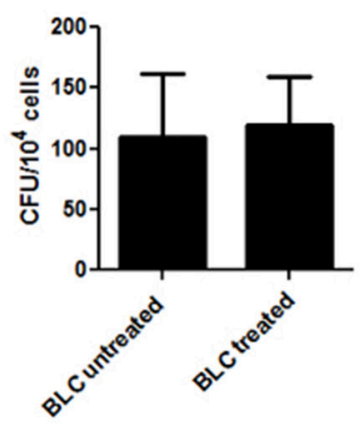

D

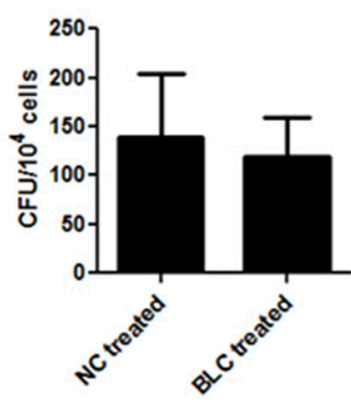

E

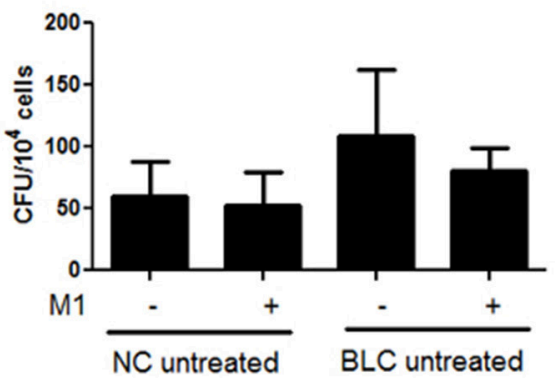

$\mathbf{F}$

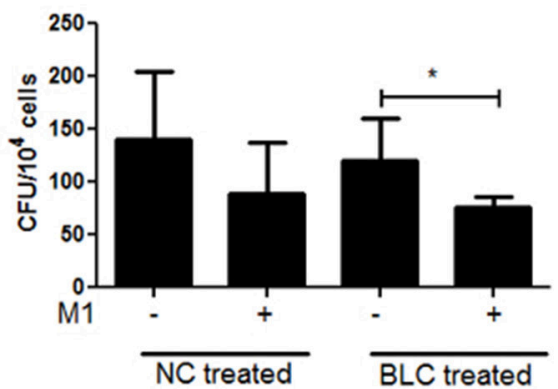

FIGURE 4 | PBMC derived macrophages from subjects with borderline risk cholesterol profiles showed reduced clearance of intracellular mycobacteria. PBMC derived macrophages were isolated from human volunteers with either normal (NC) or borderline risk (BLC) cholesterol profiles, were either untreated or cholesterol treated and then infected with M.tb H37Rv as discussed in methods. The clearance of mycobacteria by PBMC derived macrophages from the two categories was evaluated by CFU enumeration $48 \mathrm{~h}$ post-infection. Bar plots showing CFU enumeration from (A) Cholesterol untreated and treated PBMC derived macrophages of NC; (B) Cholesterol untreated PBMC derived macrophages of NC and BLC; (C) Cholesterol Untreated and treated PBMC derived macrophages of BLC; (D) Cholesterol treated PBMC derived macrophages of NC and BLC; (E) Cholesterol untreated PBMC derived macrophages of NC and BLC with or without M1 treatment and (F) Cholesterol treated PBMC derived macrophages of NC and BLC with or without M1 treatment. Each experiment was carried out at the least three times NC $(n=4)$ and BLC $(n=3)$. Statistical analyses were performed as mentioned in methods. Error bars represent $\pm S D$. ${ }^{*} P \leq 0.05 ;{ }^{*} P<0.005$.

not only the membrane organization but also mitochondriaassociated immune cell functions including infection mediated apoptosis and necrosis (Garcia-Ruiz et al., 2009). There is clinical evidence indicating familial hypercholesteremia as a risk factor for M.tb infection. Supporting the notion, administration of cholesterol-reducing drugs, such as statins is reported to control mycobacterial burden (Parihar et al., 2014). Not only mycobacteria, but treatment with lovastatin and atorvastatin reduced the growth of Salmonella enterica in murine macrophage cell line and in vivo mouse model of infection, respectively (Catron et al., 2004). Treatment with simvastatin significantly reduced the growth of Chlamydia pneumonia in mice (Erkkila et al., 2005).

Mitochondrial function is closely linked both to mitochondrial morphology and distribution, alterations in which reflect shifts in cell activity (Escobar-Henriques and Langer, 2006). Normal mitochondrial morphology is represented by networks of elongated rod-shaped mitochondria, whereas solitary, spherical or nod-shape has been linked to abnormal mitochondrial physiology in several pathological conditions (Cataldo et al., 2010). In our experiments, we found that cholesterol treated THP-1 cells had fragmented, ovoid and nod-shaped mitochondria as observed by confocal and TEM microscopy, which was more pronounced upon infection (Figures 2A,B). Heterogeneity in the mitochondrial forms was found in all conditions owing to dynamicity of fission and fusion processes. TEM images also showed the accumulated lipid bodies in cholesterol-treated cells. We experienced difficulties in staining these cells due to the presence of these lipid bodies. As shown in earlier studies (Fine-Coulson et al., 2015), we also observe that $M$.tb infection alone affected mitochondrial morphology as compared to uninfected cells (Figures 2A,B), however, mitochondria in cholesterol treated infected cells were distinctly solitary, swollen, and spherical in shape (Figure 2B lower panel, column ii) representing extreme stress that may lead to mitochondrial dysfunction.

We next correlated the alteration in mitochondrial morphology with expression of the related genes. The shape and structure of mitochondria are maintained by two opposing forces: fission and fusion (Reddy, 2009), the imbalance between the same leads to abnormal mitochondrial dynamics. Fusion and fission are controlled by evolutionarily conserved, large GTPases belonging to the family of dynamin-like MFN-1, MFN-2, OPA-1, DRP-1, and MFF. Alterations in the homeostasis 


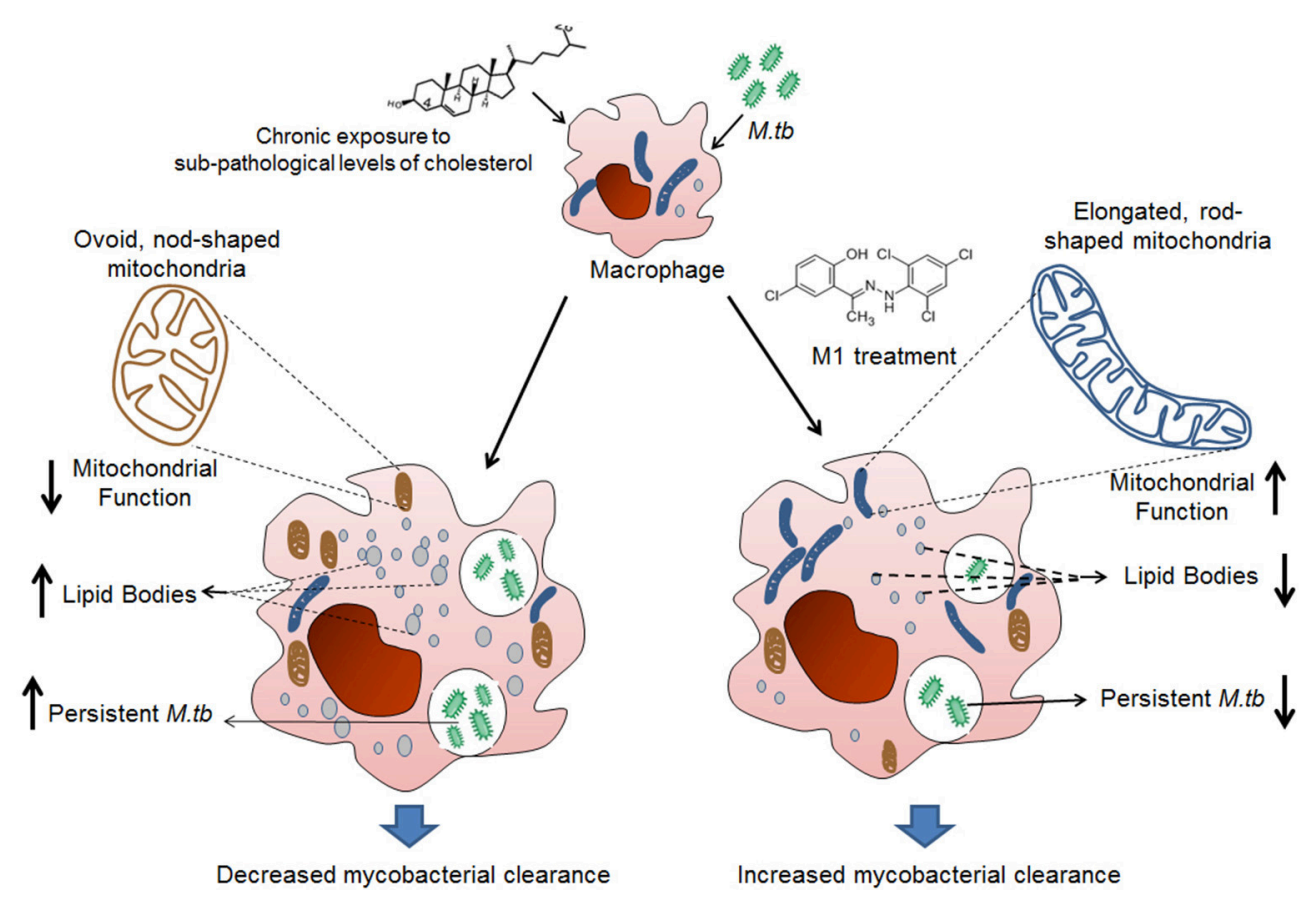

FIGURE 5 | Schematic representation of mechanism involved in M1 mediated restoration of mitochondrial function and anti-mycobacterial activity of THP-1 cells in the pre-disease model mimicking borderline risk cholesterol exposure. Prolonged exposure to sub-pathological levels of cholesterol leads to increase in intracellular lipid bodies, alteration in mitochondrial structure and function that support persistence of M.tb. Pharmacological intervention by M1 reversed changes in cholesterol treated infected macrophages, in terms of restoring structure and function of mitochondria and reducing intracellular lipid, thereby increasing the clearance of intracellular mycobacteria.

affect mitochondrial morphology and thus function. While mitochondrial fission is mediated by $d r p-1$ and $m f f$, fusion is mediated by $m f n-1, m f n-2$, and $o p a-1$. We, therefore, investigated the expression status of the structural genes $m f n-1$ and $m f n-2$, $d r p-1$, and $m f f$ by qRT-PCR. In our experiments, we observed that both $m f n-1$ and $m f n-2$ were downregulated in cholesterol treated infected cells. The reduced levels of either $m f n-1$ or $m f n-2$ cause altered mitochondrial fusion resulting in a change in mitochondrial morphology (rod-shaped mitochondria turn into nod shaped). It was interesting to note that sub-pathological cholesterol treatment alone reduced the expression of the mitochondrial fusion gene, $m f n-2$ by $47.18 \%$ (Figure 2D) and increased the expression of fission gene $d r p-1$ by $\sim 20 \%$ (Figure 2E), paving the way to mitochondrial fragmentation and therefore nod shaped structures.

Mitochondrial functional parameters are closely related to its structure, to the extent that changes in the mitochondrial reticulum due mutations of mitochondrial or nuclear-encoded genes are linked to mitochondrial oxidative phosphorylation (Zanna et al., 2008). Further, mitochondrial membranes may be coupled electrically when networked and membrane potential of individual mitochondria may change inter-dependently. We observed that MMP was reduced upon M.tb infection irrespective of cholesterol treatment (Figure 2G) indicating that infection and not cholesterol is responsible for altered MMP. In addition to changes in MMP, modifications in mitochondrial shape and distribution may be concomitant with anomalous oxidative phosphorylation and production of ATP. Testing for this possibility, we compared ATP/ADP ratios in these categories of infected cells. Like MMP, ATP/ADP, ratios were reduced equally during infection of cholesterol treated and untreated cells (Figure 2H), suggesting abnormality in ATP production as a function of infection rather than cholesterol treatment.

These changes in mitochondrial morphology, expression of the genes regulating the same and mitochondrial function could be reversed by the intervention with M1 that is known to restore mitochondrial structure (Wang et al., 2012; Figures 2). The hydrazone moiety small molecule M1 was identified by Wang et al., where it was shown through mutants (mfn-1/2 or opa-1 KOs) that M1 restores mitochondrial morphology in cells with a basal mitochondrial fusion defect. However, we observed that M1 restored levels of fission gene $m f f$ in cholesterol-treated cells. The reason behind the partial restoration of fission genes in M1 treated cells is presently unclear.

With M.tb infection prominently affecting both mitochondrial fission and fusion genes in cholesterol treated THP-1 cells, the balance of which can be restored by M1, it would be interesting to investigate the mode of action of M1 like molecule in restoring mitochondrial activity in cholesterol exposed cells. Further, M1 treatment substantially restored the mitochondrial morphology in both treated and untreated infected cells, though TEM images clearly indicated that extent of restoration of 
mitochondrial morphology was more distinct in cholesterol treated infected cells (Figures 2A,B). In terms of restoration of mitochondrial function, M1 treatment could restore MMP equally in untreated and treated infected cells (Figure 2G) but could restore $\mathrm{ATP} / \mathrm{ADP}$ ratio only in cholesterol treated infected cells (Figure 2H). We also observed that M1 treatment reduced accumulation of intracellular lipid bodies, specifically in cholesterol treated infected cells. Additionally, M1 treatment could also augment clearance of intracellular persistent M.tb more efficiently in cholesterol treated infected cells as compared to untreated infected cells (Figure 3D).

We further supported our inferences from cell culture experiments by infection assays in PBMC-derived macrophages from clinically healthy volunteers. The subjects recruited were analyzed for total serum cholesterol (TC), LDL, and HDL cholesterol and were categorized based on TC/HDL and LDL/HDL ratios. The categorization followed the global diagnostics standards as discussed earlier where those with TC/HDL ratio falling between $4.5-11$ and LDL/HDL ratio in the range 3-6 were categorized as BLC, while below this range were considered NC. We observed similar impact of $80 \mu \mathrm{M}$ treatment of cholesterol in both THP-1 model and PBMC derived macrophages from $\mathrm{NC}$ confirming the reproducibility of cell culture studies in primary cells (Figure 4A). We also observed that PBMC-derived macrophages from BLC were inefficient in clearing $\mathrm{H} 37 \mathrm{Rv}$ as compared to that by NC (Figure 4B). Interestingly, as observed in THP-1 model, M1 treatment cleared $\mathrm{H} 37 \mathrm{Rv}$ from cholesterol treated PBMC derived macrophages from BLC more effectively than untreated (Figure 4F), corroborating the observation that M1 treatment enhanced clearance of intracellular M.tb more efficiently in cholesterol exposed infected cells (Figure 3D).

TB can be prevented, and one step toward it is to protect the susceptible population. Several risk factors that have been identified which contribute to TB susceptibility and persistence including HIV infection, malnutrition, diabetes, cancer, smoking and so on (CDC, 2016). Of late, the role of lysosomal storage disorders as a susceptible factor for $\mathrm{TB}$ progression is being investigated. The mutants of lysosomal cathepsins incapacitate the lysosomes in mycobacterial clearance (Berg et al., 2016) and thereby promote mycobacterial persistence. It would also be worthwhile to investigate the role of lysosomal storage disorder upon exposure to borderline risk cholesterol and its impact on tuberculosis infection. Through this study, we establish that individuals with borderline risk cholesterol profiles, though clinically healthy, but are at the risk of susceptibility to persistent infections like that of $\mathrm{TB}$, as they are not able to respond adequately. In this study, we could show that these seemingly

\section{REFERENCES}

ATS (2000). Diagnostic standards and classification of tuberculosis in adults and children. this official statement of the American thoracic society and the centers for disease control and prevention was adopted by the ats board of directors, July 1999. this statement was endorsed by the Council of the Infectious Disease Society of America, September 1999. Am. J. Respir. Crit. Care Med. 161(4 Pt 1), 1376-1395. doi: 10.1164/ajrccm.161.4.16141 harmless levels of cholesterol could convert normal macrophages into "foamy" macrophages, caused asymptomatic damage to host cell mitochondria, which becomes apparent when M.tb infection occurred, in terms of both mitochondrial structure and function. With these perfectly viable cells failing to clear the intracellular mycobacterial load and developing susceptibility to persistent infections by non-tuberculous and attenuated mycobacteria, we propose that borderline risk cholesterol profile can be one of the factors leading to susceptibility to M.tb persistence. Systematic population studies can be designed towards validating these baseline laboratory observations to consider it as a "pre-disease" screening criterion. With the supplementation of small molecule M1 that restored mitochondrial structural and functional integrity along with increasing M.tb clearance, more efficiently in cholesterol exposed macrophages, we propose exploring such molecules as yet another strategy for host-directed therapy in TB disease management.

\section{AUTHOR CONTRIBUTIONS}

SA and SB conceived and designed the study; SA performed the experiments; SA, KM, and SB analyzed the data and wrote the manuscript. All authors have read and approved the final manuscript.

\section{FUNDING}

This study was supported by grants from Department of Science and Technology, Govt. of India (EMR/2015/001029) to SB. SA is funded by a research fellowship from ICMR, India and KM is funded by CSIR.

\section{ACKNOWLEDGMENTS}

We thank Dr. Girish and Mr. Sashikanth, NIAB, Hyderabad, India for help with confocal microscopy, Dr. Anant Patel, Mr. Harish, CCMB, and Dr. Lakshman, PVNRTVU, Rajendranagar for helping with TEM imaging. Infrastructure support by DBT-CREB, DST-FIST, DSTPURSE and UPE-II to the Department of Biochemistry and School of Life Sciences, University of Hyderabad is acknowledged.

\section{SUPPLEMENTARY MATERIAL}

The Supplementary Material for this article can be found online at: https://www.frontiersin.org/articles/10.3389/fcimb. 2017.00439/full\#supplementary-material

Almeida, P. E., Silva, A. R., Maya-Monteiro, C. M., Torocsik, D., D’Avila, H., Dezso, B., et al. (2009). Mycobacterium bovis bacillus Calmette-Guerin infection induces TLR2-dependent peroxisome proliferator-activated receptor gamma expression and activation: functions in inflammation, lipid metabolism, and pathogenesis. J. Immunol. 183, 1337-1345. doi: 10.4049/jimmunol. 0900365

Asalla, S., Girada, S. B., Kuna, R. S., Chowdhury, D., Kandagatla, B., Oruganti, S., et al. (2016). Restoring mitochondrial function: a small molecule-mediated 
approach to enhance glucose stimulated insulin secretion in cholesterol accumulated pancreatic beta cells. Sci. Rep. 6:27513. doi: 10.1038/srep,27513

Berg, R. D., Levitte, S., O’Sullivan, M. P., O’Leary, S. M., Cambier, C. J., Cameron, J., et al. (2016). Lysosomal disorders drive susceptibility to tuberculosis by compromising macrophage migration. Cell 165, 139-152. doi: 10.1016/j.cell.2016.02.034

Beutler, B. A. (1999). The role of tumor necrosis factor in health and disease. J. Rheumatol. Suppl. 57, 16-21.

Bosch, M., Mari, M., Herms, A., Fernandez, A., Fajardo, A., Kassan, A., et al. (2011). Caveolin-1 deficiency causes cholesterol-dependent mitochondrial dysfunction and apoptotic susceptibility. Curr. Biol. 21, 681-686. doi: 10.1016/j.cub.2011.03.030

BoseDasgupta, S., and Pieters, J. (2014). Striking the right balance determines TB or Not TB. Front. Immunol. 5:455. doi: 10.3389/fimmu.2014.00455

Buckman, J. F., and Reynolds, I. J. (2001). Spontaneous changes in mitochondrial membrane potential in cultured neurons. J. Neurosci. 21, 5054-5065.

CDC (2016). Available online at: https://www.cdc.gov/tb/topic/basics/risk.htm

Cataldo, A. M., McPhie, D. L., Lange, N. T., Punzell, S., Elmiligy, S., Ye, N. Z., et al. (2010). Abnormalities in mitochondrial structure in cells from patients with bipolar disorder. Am. J. Pathol. 177, 575-585. doi: 10.2353/ajpath.2010.081068

Catron, D. M., Lange, Y., Borensztajn, J., Sylvester, M. D., Jones, B. D., and Haldar, K. (2004). Salmonella enterica serovar Typhimurium requires nonsterol precursors of the cholesterol biosynthetic pathway for intracellular proliferation. Infect. Immun. 72, 1036-1042. doi: 10.1128/IAI.72.2.1036-1042.2004

Cegielski, J. P., Arab, L., and Cornoni-Huntley, J. (2012). Nutritional risk factors for tuberculosis among adults in the United States, 1971-1992. Am. J. Epidemiol. 176, 409-422. doi: 10.1093/aje/kws007

Chen, M., Gan, H., and Remold, H. G. (2006). A mechanism of virulence: virulent Mycobacterium tuberculosis strain H37Rv, but not attenuated H37Ra, causes significant mitochondrial inner membrane disruption in macrophages leading to necrosis. J. Immunol. 176, 3707-3716. doi: 10.4049/jimmunol.176.6.3707

Danelishvili, L., Everman, J., and Bermudez, L. E. (2016). Mycobacterium tuberculosis PPE68 and Rv2626c genes contribute to the host cell necrosis and bacterial escape from macrophages. Virulence 7, 23-32. doi: 10.1080/21505594.2015.1102832

Daniel, J., Maamar, H., Deb, C., Sirakova, T. D., and Kolattukudy, P. E. (2011). Mycobacterium tuberculosis uses host triacylglycerol to accumulate lipid droplets and acquires a dormancy-like phenotype in lipid-loaded macrophages. PLoS Pathog. 7:e1002093. doi: 10.1371/journal.ppat.1002093

D’Avila, H., Melo, R. C., Parreira, G. G., Werneck-Barroso, E., Castro-FariaNeto, H. C., and Bozza, P. T. (2006). Mycobacterium bovis bacillus CalmetteGuerin induces TLR2-mediated formation of lipid bodies: intracellular domains for eicosanoid synthesis in vivo. J. Immunol. 176, 3087-3097. doi: 10.4049/jimmunol.176.5.3087

Duan, L., Gan, H., Golan, D. E., and Remold, H. G. (2002). Critical role of mitochondrial damage in determining outcome of macrophage infection with Mycobacterium tuberculosis. J. Immunol. 169, 5181-5187. doi: 10.4049/jimmunol.169.9.5181

Erkkila, L., Jauhiainen, M., Laitinen, K., Haasio, K., Tiirola, T., Saikku, P., et al. (2005). Effect of simvastatin, an established lipid-lowering drug, on pulmonary Chlamydia pneumoniae infection in mice. Antimicrob. Agents Chemother. 49, 3959-3962. doi: 10.1128/AAC.49.9.3959-3962.2005

Escobar-Henriques, M., and Langer, T. (2006). Mitochondrial shaping cuts. Biochim. Biophys. Acta 1763, 422-429. doi: 10.1016/j.bbamcr.2006. 03.009

Fine-Coulson, K., Giguere, S., Quinn, F. D., and Reaves, B. J. (2015). Infection of A549 human type II epithelial cells with Mycobacterium tuberculosis induces changes in mitochondrial morphology, distribution and mass that are dependent on the early secreted antigen, ESAT-6. Microbes Infect. 17, 689-697. doi: 10.1016/j.micinf.2015.06.003

Ganji, R., Dhali, S., Rizvi, A., Sankati, S., Vemula, M. H., Mahajan, G., et al. (2016). Proteomics approach to understand reduced clearance of mycobacteria and high viral titers during HIV-mycobacteria co-infection. Cell. Microbiol. 18, 355-368. doi: $10.1111 / \mathrm{cmi} .12516$

Garcia-Ruiz, C., Mari, M., Colell, A., Morales, A., Caballero, F., Montero, J., et al. (2009). Mitochondrial cholesterol in health and disease. Histol. Histopathol. 24, 117-132. doi: 10.14670/HH-24.117
Gatfield, J., and Pieters, J. (2000). Essential role for cholesterol in entry of mycobacteria into macrophages. Science 288, 1647-1650. doi: $10.1126 /$ science. 288.5471 .1647

Goldberg, I. J. (2001). Clinical review 124: diabetic dyslipidemia: causes and consequences. J. Clin. Endocrinol. Metab. 86, 965-971. doi: $10.1210 /$ jcem.86.3.7304

Hanrahan, C. F., Golub, J. E., Mohapi, L., Tshabangu, N., Modisenyane, T., Chaisson, R. E., et al. (2010). Body mass index and risk of tuberculosis and death. AIDS 24, 1501-1508. doi: 10.1097/QAD.0b013e32833a2a4a

Hao, M., Head, W. S., Gunawardana, S. C., Hasty, A. H., and Piston, D. W. (2007). Direct effect of cholesterol on insulin secretion: a novel mechanism for pancreatic beta-cell dysfunction. Diabetes 56, 2328-2338. doi: $10.2337 / \mathrm{db} 07-0056$

Hoal, E. G. (2002). Human genetic susceptibility to tuberculosis and other mycobacterial diseases. IUBMB Life 53, 225-229. doi: 10.1080/152165402 12644

Jurcic Smith, K. L., and Lee, S. (2016). Inhibition of apoptosis by Rv2456c through Nuclear factor-kappaB extends the survival of Mycobacterium tuberculosis. Int. J. Mycobacteriol. 5, 426-436. doi: 10.1016/j.ijmyco.2016.06.018

Katsiki, N., Mikhailidis, D. P., and Mantzoros, C. S. (2016). Non-alcoholic fatty liver disease and dyslipidemia: an update. Metab. Clin. Exp. 65, 1109-1123. doi: 10.1016/j.metabol.2016.05.003

Kaul, D., Anand, P. K., and Verma, I. (2004). Cholesterol-sensor initiates $M$. tuberculosis entry into human macrophages. Mol. Cell. Biochem. 258, 219-222. doi: 10.1023/B:MCBI.0000012851.42642.be

Khan, N., Vidyarthi, A., Nadeem, S., Negi, S., Nair, G., and Agrewala, J. N. (2016). Alteration in the gut microbiota provokes susceptibility to tuberculosis. Front. Immunol. 7:529. doi: 10.3389/fimmu.2016.00529

Knight-Lozano, C. A., Young, C. G., Burow, D. L., Hu, Z. Y., Uyeminami, D., Pinkerton, K. E., et al. (2002). Cigarette smoke exposure and hypercholesterolemia increase mitochondrial damage in cardiovascular tissues. Circulation 105, 849-854. doi: 10.1161/hc0702.103977

Kronenberg, F. (2005). Dyslipidemia and nephrotic syndrome: recent advances. J. Ren. Nutr. 15, 195-203. doi: 10.1053/j.jrn.2004.10.003

Kumar, N. P., and Babu, S. (2017). Influence of diabetes mellitus on the immunity to human tuberculosis. Immunology 152, 13-24. doi: 10.1111/imm.12762

Larrouy-Maumus, G. (2015). Cholesterol acquisition by Mycobacterium tuberculosis. Virulence 6, 412-413. doi: 10.1080/21505594.2015.1053688

Lee, W., VanderVen, B. C., Fahey, R. J., and Russell, D. G. (2013). Intracellular Mycobacterium tuberculosis exploits host-derived fatty acids to limit metabolic stress. J. Biol. Chem. 288, 6788-6800. doi: 10.1074/jbc.M112.445056

Lin, P. L., Plessner, H. L., Voitenok, N. N., and Flynn, J. L. (2007). Tumor necrosis factor and tuberculosis. J. Investig. Dermatol. Symp. Proc. 12, 22-25. doi: 10.1038/sj.jidsymp. 5650027

Lonnroth, K., Castro, K. G., Chakaya, J. M., Chauhan, L. S., Floyd, K., Glaziou, P., et al. (2010). Tuberculosis control and elimination 2010-50: cure, care, and social development. Lancet 375, 1814-1829. doi: 10.1016/S0140-6736(10)60483-7

Lovewell, R. R., Sassetti, C. M., and VanderVen, B. C. (2016). Chewing the fat: lipid metabolism and homeostasis during M. tuberculosis infection. Curr. Opin. Microbiol. 29, 30-36. doi: 10.1016/j.mib.2015.10.002

Lu, X., Liu, J., Hou, F., Liu, Z., Cao, X., Seo, H., et al. (2011). Cholesterol induces pancreatic beta cell apoptosis through oxidative stress pathway. Cell Stress Chaperones 16, 539-548. doi: 10.1007/s12192-011-0265-7

Martens, G. W., Arikan, M. C., Lee, J., Ren, F., Vallerskog, T., and Kornfeld, H. (2008). Hypercholesterolemia impairs immunity to tuberculosis. Infect. Immun. 76, 3464-3472. doi: 10.1128/IAI.00037-08

Millán, J., Pintó, X., Muñoz, A., Zúñiga, M., Rubiés-Prat, J., Pallardo, L. F., et al. (2009). Lipoprotein ratios: physiological significance and clinical usefulness in cardiovascular prevention. Vasc. Health Risk Manag. 5, 757-765. doi: $10.2147 /$ vhrm.s6269

Naroeni, A., and Porte, F. (2002). Role of cholesterol and the ganglioside GM in entry and short-term survival of Brucella suis in murine macrophages. Infect. Immun. 70, 1640-1644. doi: 10.1128/IAI.70.3.1640-1644.2002

Nelson, R. H. (2013). Hyperlipidemia as a risk factor for cardiovascular disease. Prim. Care 40, 195-211. doi: 10.1016/j.pop.2012.11.003

Padmapriyadarsini, C., Ramesh Kumar, S., Terrin, N., Narendran, G., Menon, P. A., Ramachandran, G., et al. (2011). Dyslipidemia among HIV-infected Patients 
with tuberculosis taking once-daily nonnucleoside reverse-transcriptase inhibitor-based antiretroviral therapy in India. Clin. Infect. Dis. 52, 540-546. doi: $10.1093 / \mathrm{cid} / \mathrm{ciq} 195$

Panda, S. K., Kumar, S., Tupperwar, N. C., Vaidya, T., George, A., Rath, S., et al. (2012). Chitohexaose activates macrophages by alternate pathway through TLR4 and blocks endotoxemia. PLoS Pathog. 8:e1002717. doi: 10.1371 /journal.ppat.1002717

Pandey, A. K., and Sassetti, C. M. (2008). Mycobacterial persistence requires the utilization of host cholesterol. Proc. Natl. Acad. Sci. U.S.A. 105, 4376-4380. doi: 10.1073/pnas.0711159105

Parihar, S. P., Guler, R., Khutlang, R., Lang, D. M., Hurdayal, R., Mhlanga, M. M., et al. (2014). Statin therapy reduces the Mycobacterium tuberculosis burden in human macrophages and in mice by enhancing autophagy and phagosome maturation. J. Infect. Dis. 209, 754-763. doi: 10.1093/infdis/jit550

Rajapakse, N., Shimizu, K., Payne, M., and Busija, D. (2001). Isolation and characterization of intact mitochondria from neonatal rat brain. Brain Res. Brain Res. Protoc. 8, 176-183. doi: 10.1016/S1385-299X(01)00108-8

Reddy, P. H. (2009). Amyloid beta, mitochondrial structural and functional dynamics in Alzheimer's disease. Exp. Neurol. 218, 286-292. doi: 10.1016/j.expneurol.2009.03.042

Russell, D. G., Cardona, P. J., Kim, M. J., Allain, S., and Altare, F. (2009). Foamy macrophages and the progression of the human tuberculosis granuloma. Nat. Immunol. 10, 943-948. doi: 10.1038/ni.1781

Schmittgen, T. D., and Livak, K. J. (2008). Analyzing real-time PCR data by the comparative $\mathrm{C}(\mathrm{T})$ method. Nat. Protoc. 3, 1101-1108. doi: $10.1038 /$ nprot.2008.73

Vemula, M. H., Medisetti, R., Ganji, R., Jakkala, K., Sankati, S., Chatti, K., et al. (2016). Mycobacterium tuberculosis zinc metalloprotease-1 assists mycobacterial dissemination in zebrafish. Front. Microbiol. 7:1347. doi: $10.3389 /$ fmicb. 2016.01347

Viera, A. J. (2011). Predisease: when does it make sense? Epidemiol. Rev. 33, 122-134. doi: 10.1093/epirev/mxr002

Viswanathan, G., Jafurulla, M., Kumar, G. A., Raghunand, T. R., and Chattopadhyay, A. (2015). Dissecting the membrane cholesterol requirement for mycobacterial entry into host cells. Chem. Phys. Lipids 189, 19-27. doi: 10.1016/j.chemphyslip.2015.05.006

Wang, D., Wang, J., Bonamy, G. M., Meeusen, S., Brusch, R. G., Turk, C., et al. (2012). A small molecule promotes mitochondrial fusion in mammalian cells. Angew. Chem. Int. Ed Engl. 51, 9302-9305. doi: 10.1002/anie.201204589

World Health Statistics (2017). Monitoring Health for the SDGs, Sustainable Development Goals. Geneva.

Zanna, C., Ghelli, A., Porcelli, A. M., Karbowski, M., Youle, R. J., Schimpf, S., et al. (2008). OPAl mutations associated with dominant optic atrophy impair oxidative phosphorylation and mitochondrial fusion. Brain 131(Pt 2), 352-367. doi: 10.1093/brain/awm335

Conflict of Interest Statement: The authors declare that the research was conducted in the absence of any commercial or financial relationships that could be construed as a potential conflict of interest.

Copyright (c) 2017 Asalla, Mohareer and Banerjee. This is an open-access article distributed under the terms of the Creative Commons Attribution License (CC BY). The use, distribution or reproduction in other forums is permitted, provided the original author(s) or licensor are credited and that the original publication in this journal is cited, in accordance with accepted academic practice. No use, distribution or reproduction is permitted which does not comply with these terms. 